\title{
Monosynaptic Connections between Pairs of Spiny Stellate Cells in Layer 4 and Pyramidal Cells in Layer 5A Indicate That Lemniscal and Paralemniscal Afferent Pathways Converge in the Infragranular Somatosensory Cortex
}

\author{
Dirk Feldmeyer, ${ }^{1,2}$ Arnd Roth, ${ }^{2,3}$ and Bert Sakmann ${ }^{2}$ \\ ${ }^{1}$ Zelluläre Neurobiologie, Institut für Medizin, Forschungszentrum Jülich, D-52425 Jülich, Germany, ${ }^{2}$ Abteilung Zellphysiologie, Max-Planck-Institut für \\ Medizinische Forschung, D-69120 Heidelberg, Germany, and ${ }^{3}$ Wolfson Institute for Biomedical Research and Department of Physiology, University College \\ London, London WC1E 6BT, United Kingdom
}

\begin{abstract}
Monosynaptic interlaminar connections between spiny stellate cells in layer 4 (L4), the main cortical recipient layer for thalamic projections, and pyramidal cells in layer 5A (L5A), one of the main cortical output layers, were examined anatomically and functionally by paired recordings in acute brain slices. The somata of pairs forming interlaminar L4-to-L5A connections were located predominantly close to or directly under the barrel-septum wall in layer 4. Superposition of spiny stellate axon arbors and L5A pyramidal cell dendritic arbors suggested an innervation domain underneath an L4 barrel wall. Functionally, the L4-to-L5A connections were of high reliability and relatively low efficacy, with a unitary EPSP amplitude of $0.6 \mathrm{mV}$, and the connectivity was moderately high (one in seven pairs tested was connected). The EPSP amplitude was weakly depressing (paired-pulse ratio of $\approx 0.8$ ) during repetitive presynaptic action potentials at $10 \mathrm{~Hz}$. The existence of monosynaptic L4-to-L5A connections indicates that the specific "lemniscal" thalamic input from the ventrobasal nucleus of the thalamus to the cortex and the more unspecific "paralemniscal" afferent thalamic projections from the posterior medial nucleus of the thalamus merge already at an initial stage of cortical signal processing. These monosynaptic connections establish a monosynaptic coupling of the input to the cortex and its output, thereby effectively bypassing the supragranular layers.
\end{abstract}

Key words: barrel cortex; synaptic transmission; layer 4; layer 5A; cortical microcircuits; pyramidal cell

\section{Introduction}

In many sensory cortices, the complexity of receptive fields (RFs) increases successively when comparing the RFs of neurons in layers 4 (L4), L2/3, and L5 neurons, reflecting a hierarchical order of signal processing from cortical input to L4 to cortical output from L5 (Armstrong-James and Fox, 1987; Moore and Nelson, 1998). In vivo recordings of whisker-evoked subthreshold postsynaptic responses indicated a surprisingly narrow subthreshold and suprathreshold RF of L5A pyramidal cells (Manns et al., 2004). These subthreshold RFs were comparable with those of spiny neurons in layer 4, receiving direct thalamocortical input from the lemniscal pathway of the thalamus (Brecht and Sakmann, 2002). The axon arbors of L4 spiny neurons project for the most part vertically, in an almost column-restricted manner, into the supragranular layer $2 / 3$ and also to layer 5A located immediately inferior to L4 (Lübke et al., 2000, 2003). Thus, one way to

Received Dec. 22, 2004; revised Feb. 16, 2005; accepted Feb. 16, 2005.

We thank the Max-Planck Society, the Helmholtz Society, the Gatsby Charitable Foundation, and the Volkswagen Foundation for their generous financial support. We thank Moritz Helmstädter for fruitful discussions and his kind help with the axonal and dendritic density plots and Dr. Randy Bruno for comments on this manuscript.

Correspondence should be addressed to Dr. Dirk Feldmeyer, Zelluläre Neurobiologie, Institut für Medizin, Forschungszentrum Jülich, D-52425 Jülich, Germany. E-mail: d.feldmeyer@fz-juelich.de.

DOI:10.1523/JNEUROSCI.5227-04.2005

Copyright $\odot 2005$ Society for Neuroscience $\quad$ 0270-6474/05/253423-09\$15.00/0 account for the narrow RFs of L5A pyramidal cells could be that L5A pyramidal cells receive a strong direct, monosynaptic input from L4 spiny stellate cells. Whether the L4 spiny stellate cells form connections with L5A pyramidal cells is, however, an issue of debate.

L5A pyramidal cells are considered to be part of the paralemniscal sensory pathway: anterograde tracer injections into the posterior medial nucleus of the thalamus (POm) indicate that layer $5 \mathrm{~A}$ is targeted by paralemniscal afferents from this nucleus (Koralek et al., 1988; Chmielowska et al., 1989; Lu and Lin, 1993). Functional evidence indicates that the temporal response characteristics are similar for POm thalamic neurons and L5A pyramidal cells. This has been taken to suggest that these neurons are a component of the more slowly responding paralemniscal system operating in parallel to the rapidly reacting lemniscal system (Kim and Ebner, 1999; Ahissar et al., 2000, 2001). A possible integration of the lemniscal and paralemniscal inputs may then occur at the level of layer 2/3 (Ahissar et al., 2001; Ahissar and Kleinfeld, 2003).

Here we report the anatomical and functional properties of monosynaptic L4-to-L5A connections in the somatosensory cortex by making paired recordings from spiny stellate neurons in layer 4 and pyramidal cells in layer 5A in acute brain slices. We reconstructed the overlap between the $\mathrm{L} 4$ axonal and the L5A 
dendritic arbors (i.e., their innervation domains), and we determined the connectivity, the efficacy, the reliability, and the frequency-dependent modifications of these infragranular connections.

\section{Materials and Methods}

Preparation. All experimental procedures were performed according to the animal welfare guidelines of the Max-Planck Society. Wistar rats (17-23 d old) were anesthetized with isoflurane and decapitated, and coronal slices of somatosensory cortex were cut in cold extracellular solution using a vibrating microslicer (DTK-1000; Dosaka, Kyoto, Japan). Slices were cut at $350 \mu \mathrm{m}$ thickness and incubated at room temperature $\left(22-24^{\circ} \mathrm{C}\right)$ in an extracellular solution containing $4 \mathrm{~mm}$ $\mathrm{MgCl}_{2} / 1 \mathrm{mM} \mathrm{CaCl}_{2}$ to reduce synaptic activity.

Solutions. Slices were continuously superfused with an extracellular solution containing the following (in $\mathrm{mM}$ ): $125 \mathrm{NaCl}, 2.5 \mathrm{KCl}, 25$ glucose, $25 \mathrm{NaHCO}_{3}, 1.25 \mathrm{NaH}_{2} \mathrm{PO}_{4}, 2 \mathrm{CaCl}_{2}$, and $1 \mathrm{MgCl}_{2}$ (bubbled with $95 \%$ $\mathrm{O}_{2}$ and $5 \% \mathrm{CO}_{2}$ ). The composition of the pipette (intracellular) solution was as follows (in mM): $105 \mathrm{~K}$-gluconate, $30 \mathrm{KCl}, 10 \mathrm{HEPES}, 10$ phosphocreatine, 4 ATP-Mg, and $0.3 \mathrm{GTP}$ (adjusted to $\mathrm{pH} 7.3$ with $\mathrm{KOH}$ ); the osmolarity of the solution was $300 \mathrm{mOsm}$. Biocytin (Sigma, Munich, Germany) at a concentration of $3 \mathrm{mg} / \mathrm{ml}$ was routinely added to the internal solution, and cells were filled during $1-2 \mathrm{~h}$ of recording. For cell-attached stimulation (see below), we used a modified version of this solution containing the following (in $\mathrm{mM}$ ): $105 \mathrm{Na}$-gluconate, $30 \mathrm{NaCl}$, 10 HEPES, 10 phosphocreatine, 4 ATP-Mg, and 0.3 GTP (adjusted to $\mathrm{pH}$ 7.3 with $\mathrm{NaOH}$ ).

Cell identification, electrophysiological recordings, and data analysis. Slices were placed in the recording chamber under an upright microscope (fitted with $4 \times, 0.10$ numerical aperture and $60 \times$, water immersion, 1.20 numerical aperture objectives; Nikon, Düsseldorf, Germany). The barrel field was visualized at low magnification under bright-field illumination and can be identified in layer 4 as narrow dark stripes with evenly spaced, light "hollows." Individual L4 and L5A neurons were identified at $60 \times$ magnification using infrared differential interference contrast (IR-DIC) microscopy (see Fig. 1). Whole-cell voltage recordings from postsynaptic neurons were made using patch pipettes of $\sim 3.5-6$ $\mathrm{M} \Omega$ resistance pulled of thick borosilicate glass capillaries (outer diameter, $2.0 \mathrm{~mm}$; inner diameter, $0.5 \mathrm{~mm}$ ). After patching the postsynaptic L5A pyramidal cell, connections were searched using extracellular "loose-seal" stimulation in the cortical barrel (layer 4) directly above the pyramidal cell, as described previously (Feldmeyer et al., 1999, 2002). When an action potential (AP) was evoked by loose-seal stimulation, this was visible as a small deflection on the voltage trace. When the AP resulted in an EPSP in the postsynaptic L5A pyramidal cell at short latency (i.e., within $3 \mathrm{~ms}$ ), the "searching" pipette was withdrawn. The presynaptic cell was then repatched with a new recording pipette (5-7 M $\Omega$ ) filled with biocytin-containing intracellular solution, and APs were elicited in the whole-cell (current-clamp) mode. Somatic whole-cell recordings were performed at $34-36^{\circ} \mathrm{C}$. Signals were amplified using an Axoclamp amplifier for the presynaptic neuron and an Axopatch amplifier for the postsynaptic neuron (Axon Instruments, Union City, CA), filtered at $3 \mathrm{kHz}$, and sampled at $2-10 \mathrm{kHz}$ using the program Pulse (version 8.54; HEKA Elektronik, Lambrecht, Germany). Membrane potential fluctuations during current-clamp recordings were $\sim 5 \mathrm{mV}$ (peak to peak). Cell pairs in which a clear drift in the membrane potential was observed were omitted from the analysis.

Acquired data were stored on the hard disk of a Macintosh computer for off-line analysis (Igor; WaveMetrics, Lake Oswego, OR). EPSP amplitude, latency and kinetics were determined as described previously (Feldmeyer et al., 1999, 2002). All records were inspected visually. The EPSP peak amplitudes were determined within a "peak search window" of 5-8 ms after the presynaptic action potential and averaged over $0.5-1$ ms; subsequently, a baseline potential measured within a window of similar duration just preceding the EPSP was subtracted (for details, see Feldmeyer et al., 1999). Failures were defined as events with amplitudes $<1.5 \times$ the SD of the noise within the baseline window. So as not to misclassify small responses as failures, care was taken to verify that the failure average was zero. Events, the rising phase, and/or peak of which was contaminated with spontaneously occurring EPSPs were not used for analysis.

Histological procedures. After recording, slices were fixed at $4^{\circ} \mathrm{C}$ for at least $24 \mathrm{~h}$ in $100 \mathrm{~mm}$ PBS, pH 7.4, containing either 4\% paraformaldehyde or $1 \%$ paraformaldehyde and $2.5 \%$ glutaraldehyde. Slices containing biocytin-filled neurons were processed using a modified protocol described previously (Lübke et al., 2000). Slices were incubated in $0.1 \%$ Triton X-100 solution containing avidin-biotinylated horseradish peroxidase (ABC-Elite; Camon, Wiesbaden, Germany); subsequently, they were reacted using 3,3-diaminobenzidine as a chromogen under visual control until the dendritic and axonal arborization was clearly visible (usually after 2-4 $\mathrm{min}$ ). To enhance staining contrast, slices were occasionally postfixed in $0.5 \% \mathrm{OsO}_{4}(30-45 \mathrm{~min})$. Slices were then mounted on slides, embedded in Mowiol (Clariant, Sulzbach, Germany), and enclosed with a coverslip.

Biocytin-labeled pairs of neurons were examined under the light microscope at high magnification to identify putative synaptic contacts. Representative pairs were photographed at low magnification to document the dendritic and axonal arborization; potential synaptic contacts were identified as close apposition of a synaptic bouton and the postsynaptic dendrite in the same focal plane at a final magnification of $1000 \times$ ( $100 \times$ objective and $10 \times$ eyepiece) (see Fig. 2). Subsequently, neurons were reconstructed with the aid of Neurolucida software (MicroBrightField, Colchester, VT) using an Olympus Optical (Hamburg, Germany) BX50 microscope at a magnification of $1000 \times$. The reconstructions provided the basis for the quantitative morphological analysis of the following parameters: (1) location of the somata within the slice, (2) number, and (3) dendritic location of putative synaptic contacts. Corrections for shrinkage were not performed. For all data, means \pm SD are given.

Axonal and dendritic density maps. Two-dimensional (2D) maps of axonal and dendritic "length density" were constructed using the computerized 3D reconstructions (for details, see Lübke et al., 2003). The length of all axonal and dendritic branches was projected in the 2D plane and measured in a $50 \times 50 \mu \mathrm{m}$ Cartesian grid, yielding a raw density
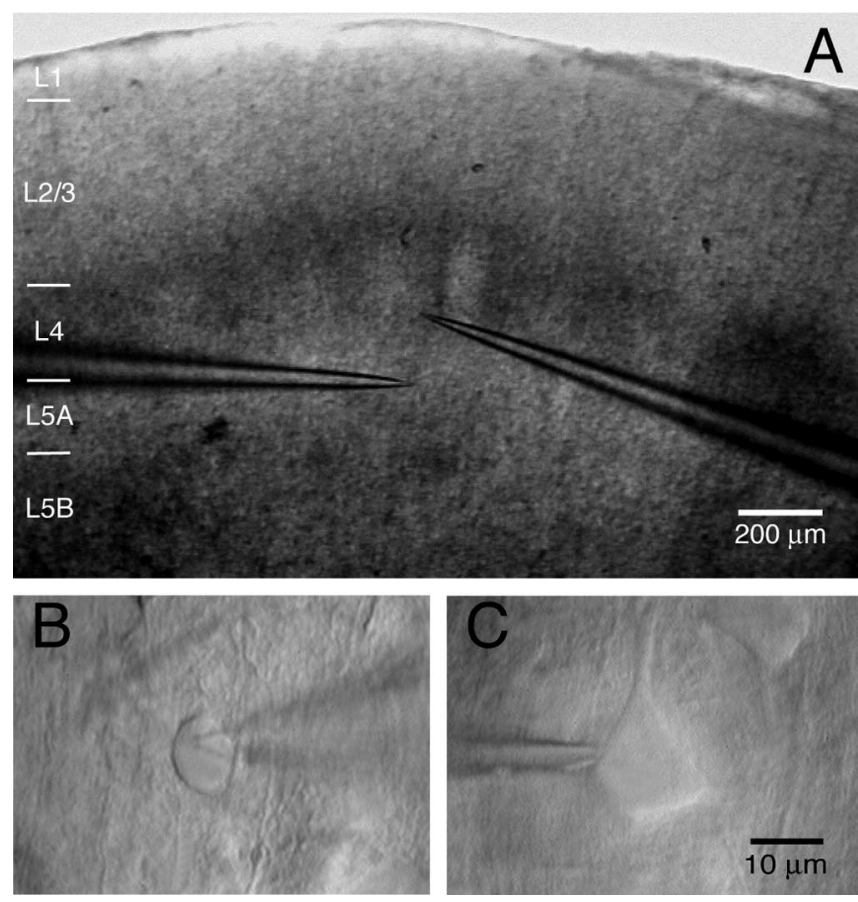

Figure 1. IR-DIC image of an L4-to-L5A connection. A, IR-DIC image of a coronal slice with clearly discernible barrels. The pipettes mark the position of the presynaptic cell (in layer 4) and the postsynaptic cell (in layer $5 \mathrm{~A}$ ). Note that both the presynaptic and the postsynaptic neurons are located at the border of the barrel column. $\boldsymbol{B}$, High-magnification image of the presynaptic spiny stellate cell. C, Postsynaptic L5A pyramidal cell. Scale bar in Calso applies to $\boldsymbol{B}$. 
A
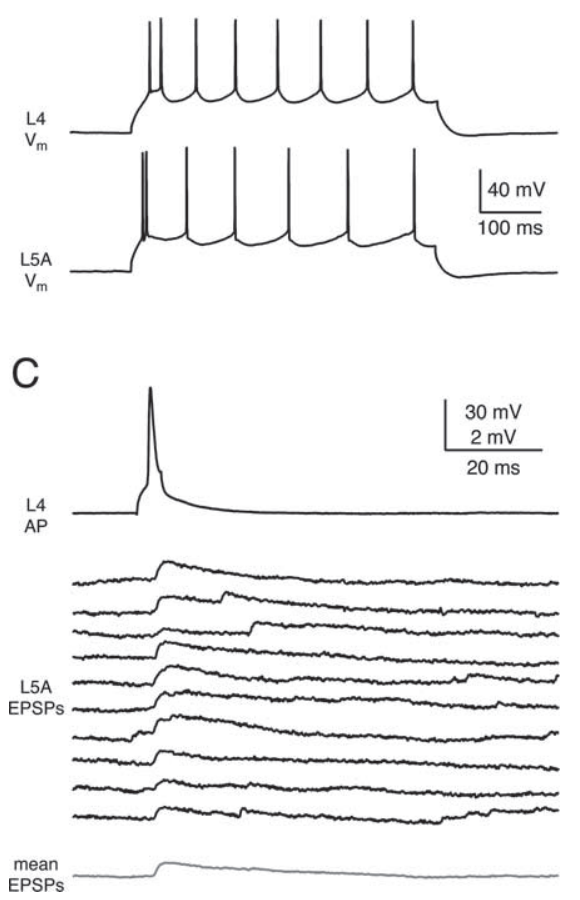

B

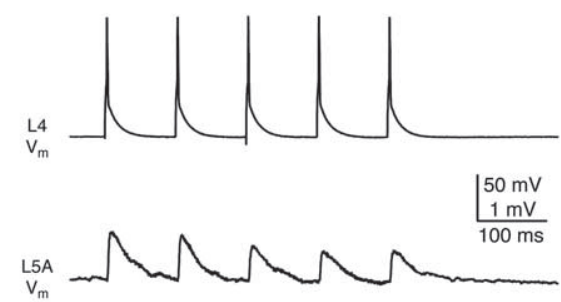

D

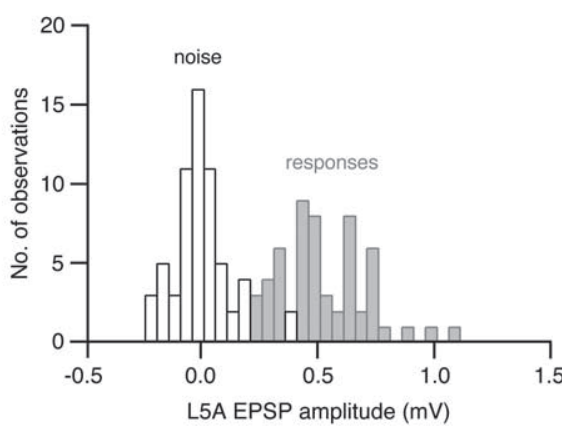

Figure 2. Reliability and efficacy of the L4-to-L5A connection. $A$, AP firing pattern of the presynaptic spiny stellate cell (top) and the postsynaptic $L 5$ pyramidal cell during current injection. Both cells display a regular firing pattern, but $L 5 A$ pyramidal cells often displayed spike doublets at the beginning of the AP train. $\boldsymbol{B}$, Short-term depression of unitary EPSPs recorded in an L5A pyramidal cell after five APs at $100 \mathrm{~ms}$ intervals in an L4 spiny stellate cell. The mean paired-pulse ratio (EPSP $/$ EPSP) was $0.83 \pm 0.08$. C, Ten consecutive EPSPs recorded in an L5A pyramidal cell elicited by APs in a presynaptic spiny stellate cell at an interstimulus interval of $15 \mathrm{~s}$. Synaptic efficacy of this connection was relatively low, but failures were not very frequent at this connection. The mean EPSP waveform (bottom trace) is shown in gray. D, EPSP amplitude distribution (gray bars) and baseline noise (white bars) for the same synaptic connection as shown in $\boldsymbol{C}$.

map. For alignment of these maps with respect to the barrel center, barrel borders were identified in the low power $(2.5 \times$ objective $)$ bright-field micrographs made from the acute brain slice (Lübke et al., 2000, 2003; Feldmeyer et al., 2002). Spatial low-pass filtering of these maps was performed by $2 \mathrm{D}$ convolution with a Gaussian kernel $(\sigma=50 \mu \mathrm{m})$, and continuous $2 \mathrm{D}$ density functions were constructed using bicubic interpolation in Mathematica 4.1 (Wolfram Research, Champaign, IL). The axonal and the dendritic length density maps thus obtained were then multiplied to calculate the predicted "innervation domain" between L4 spiny neurons and L5A pyramidal cells (Lübke et al., 2003).

\section{Results}

Visualization of spiny stellate and pyramidal cells in L4 and L5A in the acute coronal slice preparation

Figure 1 shows an acute coronal slice (Fig. $1 A$ ) and the somata of an $\mathrm{L} 4$ spiny neuron (Fig. $1 B$ ) and an L5A pyramidal cell (Fig. $1 C$ ) of the barrel cortex. The tips of two independent whole-cell recording pipettes are attached to the somata that are located within the outline of a barrel (layer 4) and underneath a barrel (layer 5A). Such pairs of synaptically connected cells were found only when L4 and L5A neurons were located very close to the barrel wall (within $\sim 50 \mu \mathrm{m}$ from the barrel border). Indeed, it has been suggested previously that L5 pyramidal cells are in preferential register with the walls and septa of the barrels (Crandall et al., 1986). The average vertical distance was $164 \pm 47 \mu \mathrm{m}$ between the two cell somata ( $n=12$ pairs). An important parameter for the reconstruction of cortical circuitry is the connectivity of intralaminar and interlaminar connections between specific cell types, which is the probability of finding connected pairs of neurons. The L4-to-L5A connections are rather stereotyped in their location within a column, and we found a connectivity of $0.14 \pm 0.10$ (i.e., of seven trials of paired recordings, only one recording was from connected cells; not included are L5A pyramidal cells for which no connections were established).

L4-to-L5A pairs: reliability, efficacy, and short-term modification

Both L4 spiny stellate cells and L5A pyramidal cells show regular AP firing patterns (Fig. 2A) during depolarizing current injections. In addition, L5A pyramidal cells usually showed an AP doublet at the beginning of the AP train. After an L4-toL5A connection was established, APs were elicited in the L4 neuron by brief current injection at 10-20 s intervals. These APs elicited unitary EPSPs of small and variable amplitude in L5A pyramidal cells. EPSP characteristics were determined from series of 50-180 EPSPs (Fig. 2C,D). In 12 individual connections, the EPSP amplitude varied between 0.13 and $1.12 \mathrm{mV}$; the average amplitude was $0.61 \pm 0.40 \mathrm{mV}$. The EPSPs rose with a $20-80 \%$ rise time of $1.1 \pm$ $0.5 \mathrm{~ms}$ and decayed with a time constant of $13.8 \pm 5.1 \mathrm{~ms}(n=$ 12). Figure $2 C$ illustrates successive unitary EPSPs elicited by APs in the presynaptic L4 spiny stellate cell. The connections are characterized by infrequent failures (mean failure rate, $8.3 \% \pm 10.2 \%$ ), and the failure rate did not exceed $30 \%$, even when the EPSP amplitude was small $(<0.2 \mathrm{mV})$. In accordance with this, the average coefficient of variation was also low $(0.33 \pm$ 0.20 ), indicating a relatively high release probability. Physiological parameters obtained from postsynaptic L5A pyramidal cells located underneath a septum or a barrel were not significantly different ( $t$ test; $p>0.2$ ). We also tested whether the L4-to-L5A connections were reciprocal, i.e., whether APs in the L5A pyramidal cell elicited EPSPs; however, this was not the case.

The unitary EPSP amplitude of the L4-to-L5A connection

Table 1. Comparison of properties of synaptic connections established by L4 spiny neuron nerve terminals projecting onto other excitatory neurons in different cortical layers

\begin{tabular}{llcc}
\hline L4 projection to: & L4 spiny stellate & L2/3 pyramidal cells & L5A pyramidal cells \\
\hline Unitary EPSP $(\mathrm{mV})$ & $1.6 \pm 1.5(n=131)$ & $0.7 \pm 0.6(n=64)$ & $0.6 \pm 0.4(n=12)$ \\
Coefficient of variation & $0.37 \pm 0.18(n=131)$ & $0.27 \pm 0.13(n=63)$ & $0.33 \pm 0.20(n=12)$ \\
Paired-pulse ratio (100 ms interstimulus interval) & $0.73 \pm 0.25(n=8)$ & $0.87 \pm 0.31(n=7)$ & $0.83 \pm 0.08(n=4)$ \\
Connectivity & $0.2-0.3$ & 0.1 & 0.1 \\
\hline
\end{tabular}

This table includes data from Feldmeyer et al. (1999, 2002). 

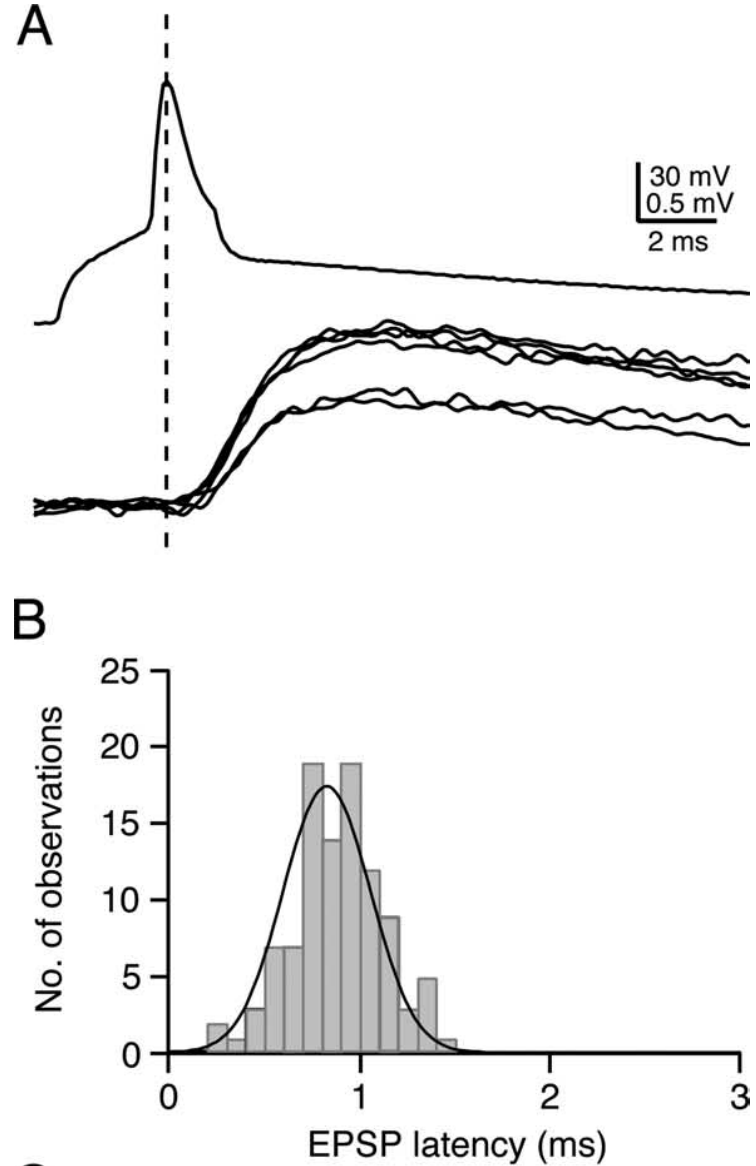

C

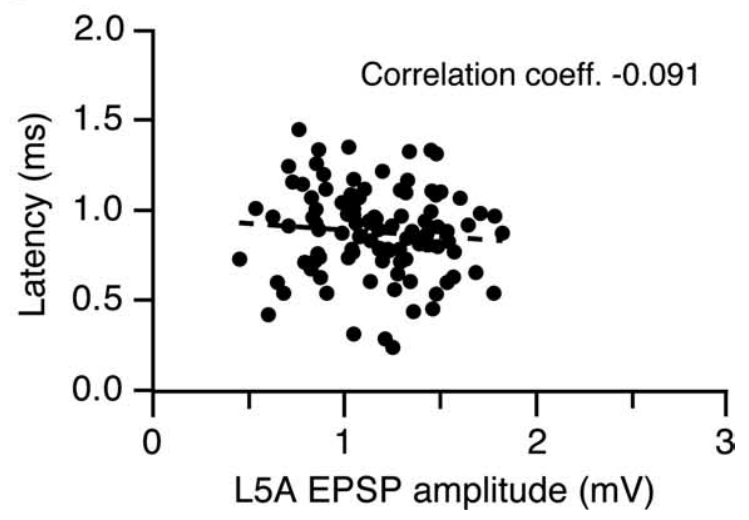

Figure 3. EPSP latency at the L4-to-L5A connection. $A$, Presynaptic AP (top trace) and unitary EPSPs (bottom traces) recorded from a synaptic connection between an L4 spiny stellate and an L5A pyramidal cell. $\boldsymbol{B}$, Latency distribution of unitary EPSPS, determined between the peak of the presynaptic AP and the onset of the EPSP. Note that the distribution of latencies for a single connection is rather broad (half-width of $0.53 \mathrm{~ms}$ ), suggesting different electrotonic distances between synaptic contacts. C, Plot of latencies against the EPSP amplitude. There was a slight but statistically nonsignificant decrease in EPSP latency with increasing unitary EPSP amplitude (correlation coefficient, -0.091 ).

depresses after stimulation by repeated APs (Fig. $2 B$ ) in very much the same way as unitary EPSPs at the L4-to-L2/3 and the L4-to-L4 connection made by layer 4 intralaminar and by ascending interlaminar collaterals of the spiny stellate cell axons (Feldmeyer et al., 1999, 2002) (Table 1). For an interpulse interval of $100 \mathrm{~ms}$, the paired-pulse ratio in these connections is $0.83 \pm$ 0.08 , a value that is not significantly different from other synaptic connections in which L4 spiny neurons are presynaptic (Table 1).
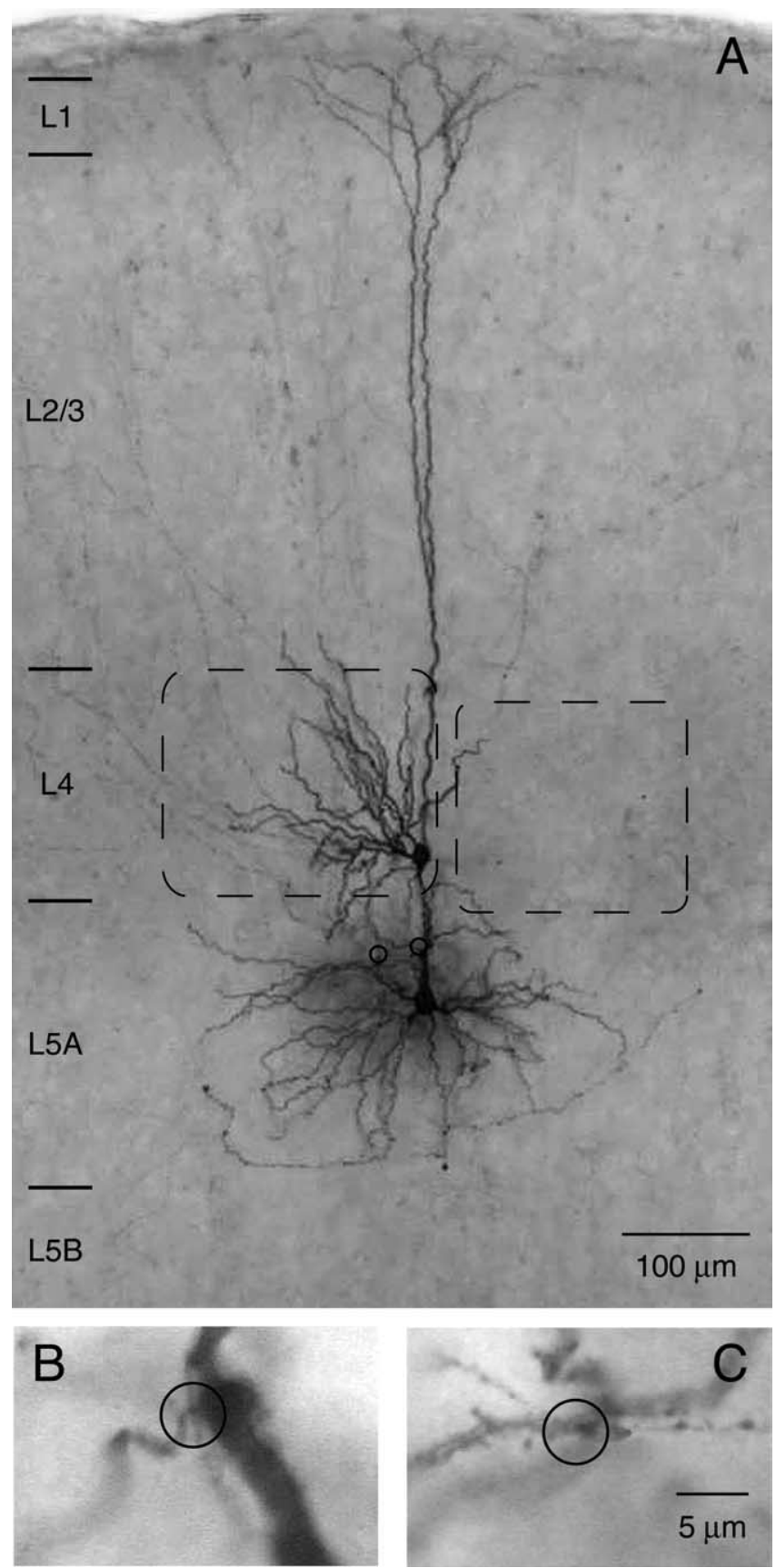

Figure 4. Biocytin staining of an $\mathrm{L} 4$ spiny stellate-L5A pyramidal cell pair. A, Half-tone photomicrograph of a biocytin-filled pair between a spiny stellate cell in layer 4 and a pyramidal cell in layer $5 \mathrm{~A}$. Note that apical oblique dendrites are strictly confined to layer $5 \mathrm{~A}$ and lower layer 4 and that the field span of the apical tuft is smaller than that of the basal dendritic tree. Barrels are indicated by dashed lines. Two light-microscopically identified putative synaptic contacts are marked by open circles. $\boldsymbol{B}, \boldsymbol{C}$, Higher magnification of the two putative synaptic contacts. Both synaptic contacts were established on apical oblique dendrites. Scale bar in $\boldsymbol{C}$ also applies to $\boldsymbol{B}$.

This indicates that the degree of depression in stellate cell connections is projection (presynaptic) cell specific, at least insofar as connections with other excitatory neurons are concerned.

\section{L4-to-L5A pairs: EPSP latency}

Figure $3 A$ illustrates the time course of APs evoked by current injection in the soma of a presynaptic L4 spiny stellate cell (top 


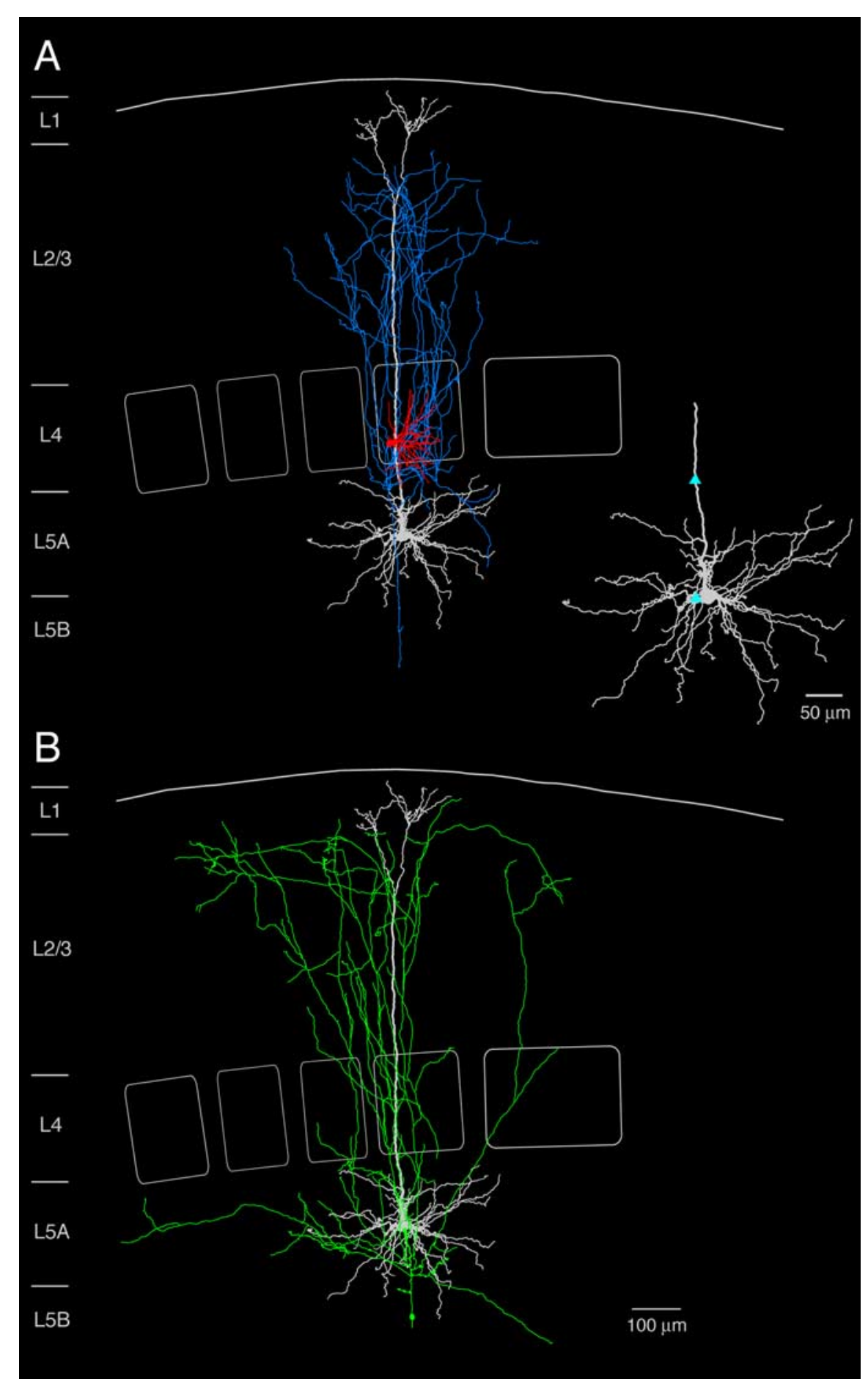

Figure 5. Reconstruction of an L4-to-L5A pair. A, Neurolucida reconstruction and 2D projection of another synaptically connected pair of an $\mathrm{L} 4$ spiny stellate cell and an $\mathrm{L} 5$ pyramidal cell. The axonal arbor of the spiny stellate cell (blue) and the dendritic arbor of the spiny stellate cell (red) and the postsynaptic L5A pyramidal cell (white) are shown in $\boldsymbol{A}$. Inset, Dendritic domain of the postsynaptic L5A pyramidal cell; putative synaptic contacts are marked by light-blue triangles. $\boldsymbol{B}$, Axonal arborization of the postsynaptic L5A pyramidal cell. Note that L5A pyramidal cells have prominent vertical axonal collaterals projecting up to the layer 1 border. Here, axon collaterals are oriented in the horizontal direction.

trace) and that elicited unitary EPSPs in a postsynaptic L5A pyramidal cell (bottom trace). Successive unitary EPSPs displayed variable amplitudes and variable latencies, as is also apparent in the broad latency distribution (Fig. 3B). The latency is independent of the EPSP amplitude (Fig. 3C). On average, L4-to-L5A connections show a rather short latency of $1.0 \pm 0.1 \mathrm{~ms}(n=7$; cells with poor presynaptic $R_{\text {access }}$ or poor latency fits were excluded from the latency analysis), with only small variability between connections (0.9-1.2 ms). However, unitary EPSPs at synapses between L4 spiny stellate cells and L5A pyramidal cells show a larger degree of latency variation within a given connection than L4-to-L4 and L4-to-L2/3 connections (Feldmeyer et al., 1999, 2002). This may imply that synaptic contacts between L4 spiny stellate cells and L5A pyramidal cells are more distributed and electrotonically more distant with respect to the soma.

\section{Axonal and dendritic overlap of L4-to-L5A pairs}

After whole-cell recording of cell pairs, both neurons were loaded with biocytin, fixed, and subsequently visualized, and their dendritic and axonal arbors were reconstructed. Five pairs of neurons were sufficiently well filled to allow a complete anatomical reconstruction and lightmicroscopic identification of putative synaptic contacts. Figure $4 A$ illustrates the axonal and dendritic arborization of a pair of an L4 spiny stellate cell and an L5A pyramidal cell that were located, with their somata, close to the border between the two layers. All connected pairs of L4 spiny stellate and L5A pyramidal cells were located at the lateral border of a barrel column (Figs. 5A, 6A). Some of the postsynaptic L5A pyramidal cells were located within the barrel column ( $n=7$ of 12$)$, and a smaller fraction was located in the septum between two adjacent barrel columns $(n=5)$. L4 spiny stellate cells were unequivocally identified by their highly asymmetric dendritic arbors facing the barrel hollow. L5A pyramidal cells had long and thin apical dendrites projecting to layer 1 , in which they formed small apical tufts branching mostly in layer 1; apical oblique dendrites are generally restricted to layer 5A and lower layer 4. Similarly, their basal dendritic arbor was confined to layer 5 (Manns et al., 2004).

Figure $6 \mathrm{~A}$ shows the $2 \mathrm{D}$ projection of $3 \mathrm{D}$ reconstructions of the dendritic and axonal arbors of the L4 spiny stellate cell and the dendritic arbor of the L5A pyramidal cell pairs to illustrate the overlap of L4 axonal arbors and L5A dendritic arbors. The axonal arbor of L5A pyramidal cells was less restricted than that of L4 spiny neurons and projected into adjacent columns at the level of layers 5,4 , and $2 / 3$ (Figs. 5B, 6B). There was a prominent axonal domain within layer $5 \mathrm{~A}$ itself, suggesting a high connectivity within this layer. Numerous collaterals ascended to layers 4 and 2/3. These collat- 

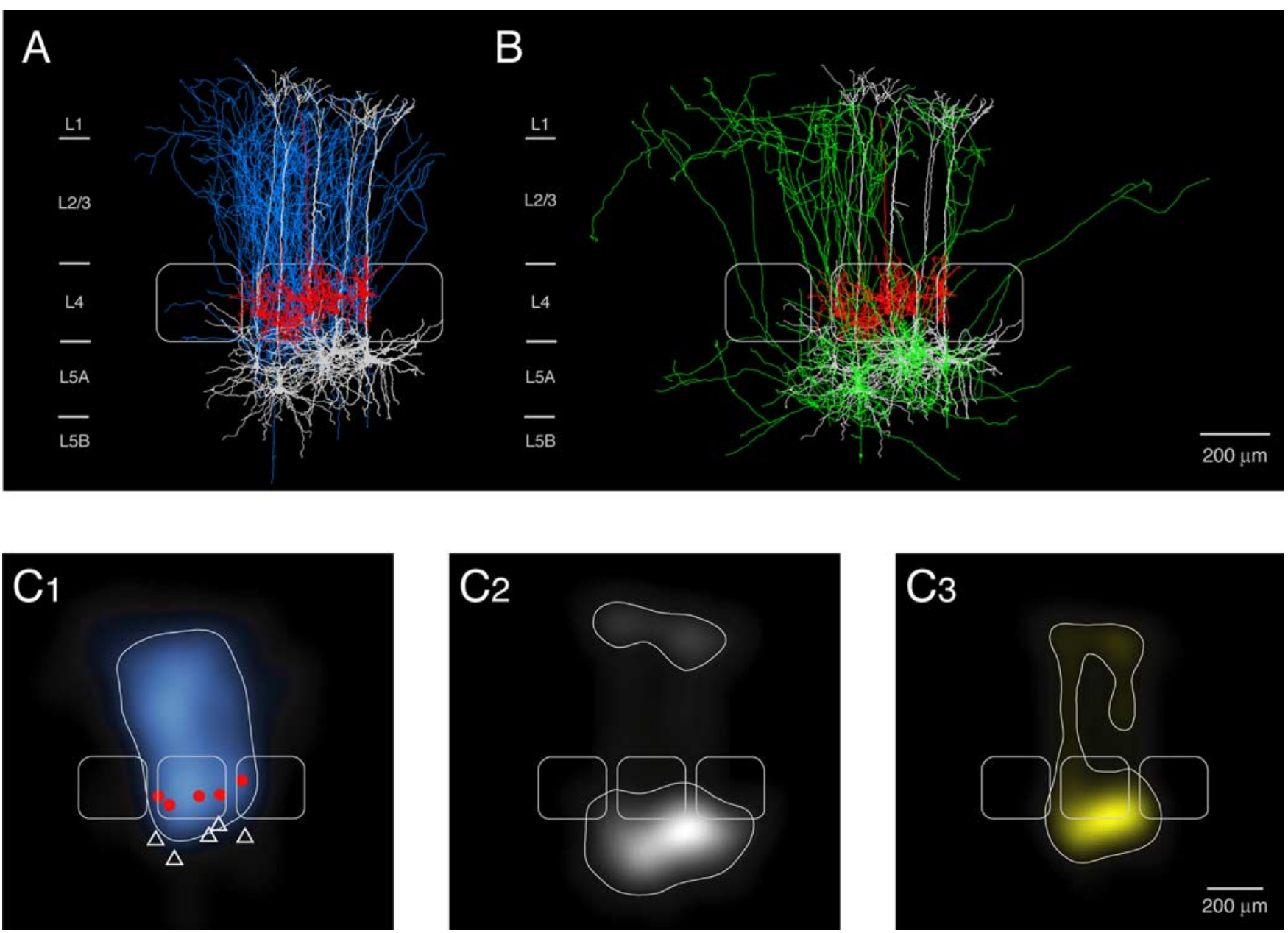

Figure 6. Overlay and density maps of L4-to-L5A connections. A, Barrel-centered overlay of five synaptically coupled L4-to-L5A pairs. Connections were found almost exclusively between neurons at the border of the barrel column; however, the postsynaptic LSA pyramidal cell was generally located right below the barrel in layer 4 . The presynaptic neurons usually had a highly asymmetric dendritic tree. The average barrel in the center is outlined in white; two neighboring barrels are added symbolically. $\boldsymbol{B}$, Superposition of Neurolucida reconstructions of the postsynaptic L5A pyramidal cell axons from the same L4-L5A pairs as in $\boldsymbol{A}$, aligned with respect to the barrel center. The dendritic domain of the postsynaptic L5A pyramidal cells (white) and that of the presynaptic L4 spiny neurons (red) is also shown. Same barrels as in $\boldsymbol{A}$. Note that the $L 5 A$ axon projects considerably into adjacent barrel columns. C, $2 D$ maps of axonal (C1) and dendritic (C2) length density of synaptically coupled L 4 spiny neurons and L 5 A pyramidal cells, respectively. L4 spiny neurons and L5A pyramidal cells $(n=5)$ were aligned with respect to the center of the barrel. The predicted innervation domain (C3) of L5A dendrites by $L 4$ axons is given by the product of the $L 4$ axonal density and the L5A dendritic density. Contours (thin white lines) enclosing $80 \%$ of the integrated density are shown superimposed. Positions of L4 spiny neuron somata (red dots), L5A pyramidal cell somata (white triangles), and outlines of barrels (thicker white lines) are indicated symbolically.

erals displayed an extensive arborization at the border between layer 1 and layer 2/3, and collaterals running parallel to the border between these layers (up to several $100 \mu \mathrm{m}$ ) were frequently observed (see also Fig. $8 \mathrm{~A}$ ).

Figure $6 C$ illustrates quantitatively the overlap of L4 axonal arbors (Fig. 6C1) and L5A dendritic arbors (Fig. 6C2), suggesting that potential synaptic contacts might be located on both the basal and apical dendrites of L5A pyramidal cells (Fig. 6C3). L4to-L5A connections established between two and four (2.4 \pm 0.9 ; $n=5$ pairs) putative synaptic contacts, which were predominantly located on the apical oblique dendrites located in layer $5 \mathrm{~A}$ and lower layer 4 (Figs. $4 B, C, 5 A$, inset). However, contacts were also made with L5A basal dendrites (Fig. 5A, inset), and one contact was found on the apical tuft of the L5A pyramidal cell. Figure $7 A$ illustrates schematically the layer 4 inputs to L5A pyramidal cells as well as to $\mathrm{L} 4$ spiny neurons and to $\mathrm{L} 2 / 3$ pyramidal cells. The outlines of the three respective innervation domains (Fig. 7B) demonstrate that all target regions of L4 spiny neurons are essentially columnar in nature.
Axon arbors of L5A pyramidal cells in supragranular layers Finally, by superimposing the axonal arbors of L5A pyramidal cells from five pairs with the dendritic arbors of L2/3 pyramidal cells (pooled data from Lübke et al., 2003) (D. Feldmeyer, J. Lübke, and B. Sakmann, unpublished data), it becomes obvious that L5A axon collaterals project laterally mostly in L5A and vertically into layer $2 / 3$ (Fig. $8 A, B$ ). Significantly, the axon collaterals arborise laterally close to the layer 1 border, suggesting that they innervate the apical tufts of L2/3 and L5 pyramidal cells of the same and other (L2/3) cortical laminas.

The prospective innervation domain of L5A-to-L2/3 connections is shown in Figure $8 C$. The outline of the innervation domain of L5A-to-L2/3 connections indicates that L5A cells can excite $\mathrm{L} 2 / 3$ cells via their basal and their apical dendrites.

\section{Discussion}

Granular layer 4 is the main recipient layer of the cortex, whereas layer $5 \mathrm{~A}$ is a main output layer, respectively. Because both layers are connected monosynaptically by "reliable" synapses, the sen- 
A
Pia

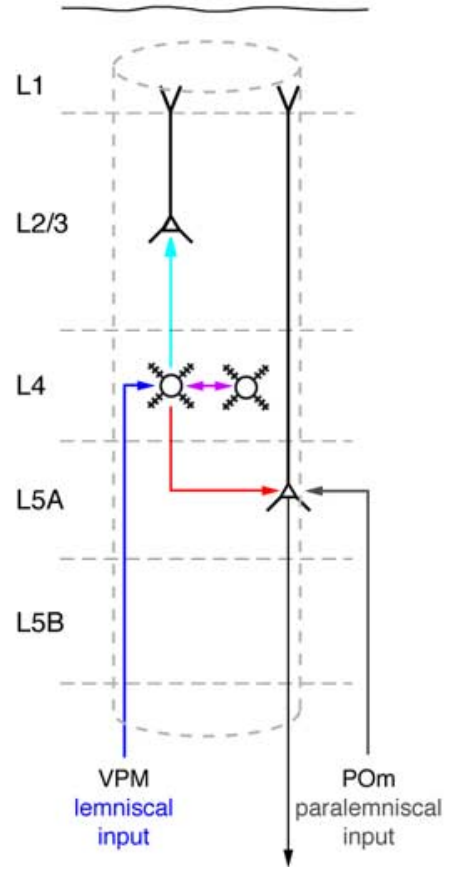

Pia

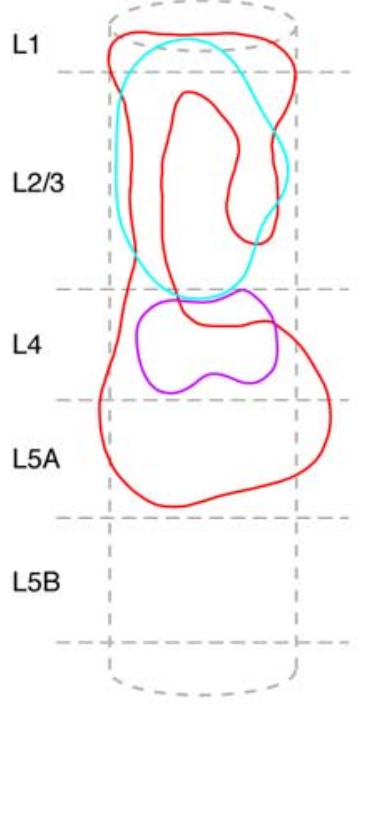

Figure 7. L4 spiny neuron connections and their innervation domains. $\boldsymbol{A}$, Schematic representation of the projections of L4 spiny stellate neurons to three different layers (violet, lightblue, and red arrows) and of the infragranular convergence of the lemniscal (dark blue) and paralemniscal (gray) afferent pathway in L5A pyramidal cells. $\boldsymbol{B}$, Innervation domains (80\% contour lines) for the three types of excitatory synaptic connections in which $L 4$ spiny neurons act as presynaptic (sender) neurons. Violet, L4-to-L4 connection; light blue, L4-to-L2/3 connection; red, L4-to-L5A connection. Note the similar horizontal extent of all three innervation domains, which suggests that they are confined to a cortical column.

sory excitation arriving via the lemniscal [ventral posterior medial nucleus (VPM)-to-L4] and the paralemniscal (POm-toL5A) afferents converge at least partially, in infragranular layers. The L4-to-L5A connections may form a "short circuit" between afferent signals to the cortex and efferent signals that leave the cortex from layer $5 \mathrm{~A}$ and project to subcortical regions, for ex- ample, the basal ganglia (Mercier et al., 1990; Alloway et al., 1999). The short circuit has the effect that afferent excitation at least potentially bypasses the supragranular layers (layers $2 / 3$ and 1), as illustrated schematically in Figure $7 A$.

\section{Projection cell specificity of synaptic short-term modification in $\mathrm{L} 4$ axons}

We previously characterized the L4-to-L4 connections and the L4-to-L2/3 connections of spiny stellate cells. The innervation domains of these connections are less extensive (Fig. 7B). However, the properties of unitary EPSPs are comparable (Table 1). Comparison of the paired-pulse ratio of spiny stellate terminals projecting to $\mathrm{L} 2 / 3$ and to $\mathrm{L} 4$ neurons with the short-term changes in terminals of connections with layer 5A (Fig. 6A) shows that short-term modulation is not very different in these three innervation domains of L4 spiny neurons (Table 1). This similarity suggests that short-term modification of release from L4 spiny stellate boutons is predominantly "projection-cell" specific, in contrast to boutons of L2/3 pyramidal cell axons in which the short-term modulation of release is "target-cell" specific, because the paired-pulse ratio varies with the type of neuron contacted (Markram et al., 1998; Reyes et al., 1998; Rozov et al., 2001).

\section{Output of L4 establishes a "shortcut" between lemniscal and paralemniscal pathways}

The L5A pyramidal cells are excited by thalamic afferents from the POm (Chmielowska et al., 1989; Lu and Lin, 1993; Ahissar et al., 2000, 2001) and, in addition, by intracortical inputs from layer 4 described here as well as from layer $2 / 3$ and layer $5 \mathrm{~A}$ (Feldmeyer, unpublished observation). Thus, the excitation conveyed by lemniscal and paralemniscal thalamic projections, also termed "specific" and "unspecific," is merging in L5A, as shown schematically in Figure 7, $A$ and $B$. This convergence could enhance the excitation of the output of the cortex via L5A pyramidal cells by attention-related nonsomatosensory signals. Here we show that specific lemniscal excitation of L4 spiny stellates is conveyed monosynaptically to the apical oblique and basal dendrites of L5A pyramidal cells. Our data are supported by recent studies using multielectrode arrays or flash photolysis of caged glutamate, from which input to L5A pyramidal cells from layer 4 can be inferred (Schubert et al., 2003; Wirth and Lüscher, 2004).
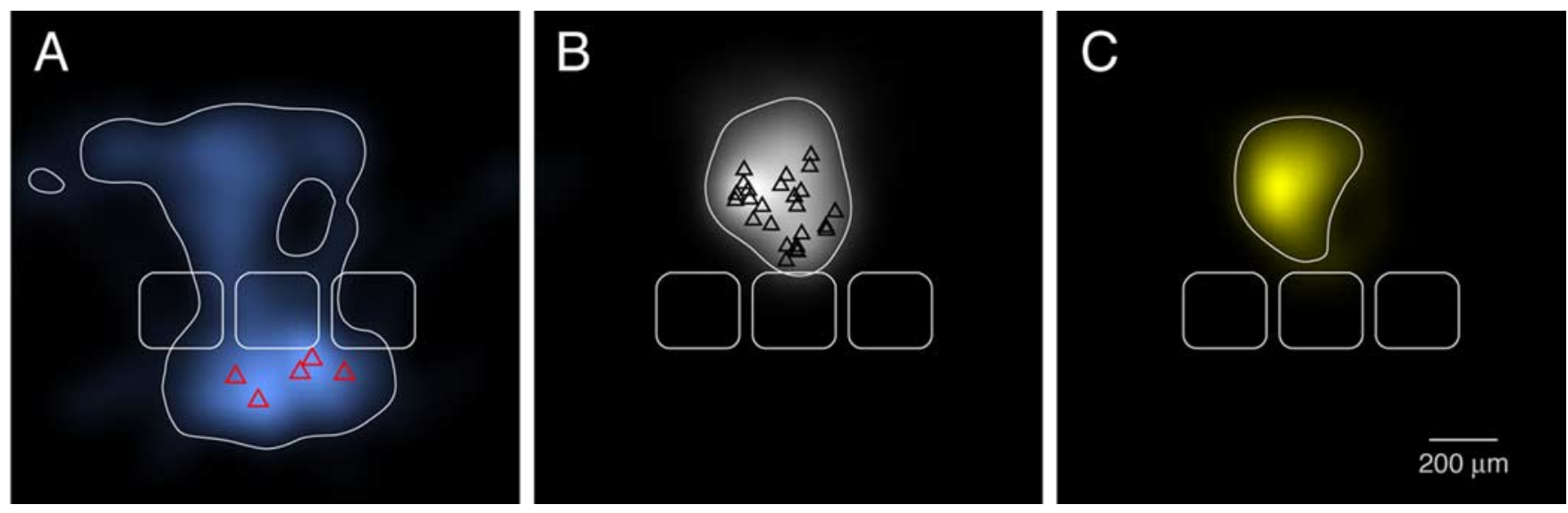

Figure 8. $L 5 A-t o-L 2 / 3$ connections and their prospective innervation domains. $A, B, 2 D$ maps of axonal length density of $L 5 A$ pyramidal cells ( $A$, blue; $L 5 A$ pyramidal somata in red) and dendritic length density of $L 2 / 3$ pyramidal cells ( $\boldsymbol{B}$, white; $L 2 / 3$ pyramidal somata in black). For the $L 2 / 3$ pyramidal cell dendrites, data to generate length density maps were pooled from $L 4$-to- $L 2 / 3$ connections (Lübke et al., 2003) and from L2/3-to-L2/3 connections (Feldmeyer, Lübke, and Sakmann, unpublished data). C, Innervation domain (yellow) of L5A axons projecting to L2/3 dendrites, suggesting the existence of a bidirectional translaminar connection between these two layers. The contour lines (white) on the $2 \mathrm{D}$ map include $80 \%$ of the integrated density. Average barrels are taken from Figure 6 . 
When the sensory inputs from the VPM are combined with a more unspecific excitation from the POm afferents that synapse also onto the basal and on apical dendrites of L5A pyramidal cells, then near-coincident input from the two thalamocortical projections could generate an output pattern of APs in L5A pyramidal cells that is almost independent of the excitation of the supragranular layers 2 and 3. Interestingly, in rodents, the ablation of layer $2 / 3$ seems to affect navigation tasks by somatosensory cues only little, but only when acquired before ablation [M. H. Friedberg, S. M. Lee, and F. F. Ebner, unpublished data cited in Huang et al., (1998)].

However, L5A pyramidal cells also receive synaptic input via monosynaptic L2/3-to-L5A connections (Feldmeyer, unpublished observation). The question arises which input to L5A pyramidal cells dominates the output of these cells with respect to stimulus-related timing and strength. This can be tentatively answered by comparing the latency of different inputs to layer $5 \mathrm{~A}$.

\section{Latency of responses in L5A pyramidal cells}

In vitro, an AP in the L4-to-L5A pathway evokes an EPSP with $\sim 1.0$ ms latency, whereas the layer 4 input to L2/3 pyramidal cells has a latency of $1.6 \mathrm{~ms}$ (Feldmeyer et al., 2002). Correspondingly, in vivo, the onset latency of EPSPs evoked by a principal whisker deflection in an L5A pyramidal cell is comparable with that evoked in barrel-related L2/3 pyramidal cells (10.1 and $9.6 \mathrm{~ms}$, respectively) (Brecht et al., 2003; Manns et al., 2004). This means that the L2/3-to-L5A input will be activated later and with less synchrony because APs in layer 2/3 arise from EPSPs with a delay of at least a few and up to tens of milliseconds (Brecht et al., 2003). Finally, the L2/3-to-L5A pathway introduces an additional delay of at least $1 \mathrm{~ms}$. Thus, it is likely that the earliest input to L5A cells is provided by L4 spiny neurons, which is then followed by an asynchronous $\mathrm{L} 2 / 3$ input. The paralemniscal input to layer $5 \mathrm{~A}$ is active even later, at least under conditions of anesthesia (Ahissar et al., 2000, 2001). Whisker responses in septum-related neurons receiving direct paralemniscal input were found to have a clearly longer latency than those in L2/3 pyramidal cells receiving lemniscal input (23 vs $10 \mathrm{~ms}$ ) (Brecht et al., 2003). Together, the latencies of different pathways converging on L5A pyramidal cells (L4-to-L5A, L2/3-to-L5A, and POm-to-L5A and possibly feedforward input from L5A cells) suggest that the earliest input to layer $5 \mathrm{~A}$ during whisker deflection is dominated by layer 4 . This input may not be purely excitatory because various types of L4 interneurons receive also direct monosynaptic input from the VPM (Porter et al., 2001; Swadlow, 2003) and thus may affect the excitatory signal flow through feedforward inhibition. In addition, feedback inhibitory mechanisms may shape the response of excitatory neurons in the barrel cortex (Zhu et al., 2004).

Tracer studies and functional evidence suggest that the paralemniscal thalamocortical afferents project to layer $5 \mathrm{~A}$, the septa in layer 4, and layer 1 (Koralek et al., 1988; Chmielowska et al., 1989; Lu and Lin, 1993; Kim and Ebner, 1999; Ahissar and Kleinfeld, 2003). Activation of pyramidal cells via layer 1 afferents is thought to mediate changes in sensory attention (Cauller and Kulics, 1991). The L5A pyramidal cells could thus act as detectors for near-coincident lemniscal (VPM-to-L4 followed by L4-toL5A input to their basal and oblique dendrites) and paralemniscal input (POm-to-L5A input to their basal and apical tuft dendrites) when the animal is predominantly navigating via somatosensory signals.

\section{Feedback loops in the neocortex}

Feedback circuits are a common feature of the neocortex. A well known example is that between the lemniscal thalamic afferents and a subset of L6 pyramidal cells. Whereas VPM thalamic neurons project to both layers 4 and 6 [and the thalamic reticular nucleus (nRT)], L6 pyramidal cell project back to the VPM and the nRT (for review, see Castro-Alamancos, 2004). L6 pyramidal cells are also involved in another feedback circuit, that between layers 4 and 6. This circuit has been identified indirectly in the somatosensory cortex (Wirth and Lüscher, 2004), but paired recordings showed its existence in the visual cortex (TarczyHornoch et al., 1999).

The geometry of L2/3 and L5A pyramidal cell axon arbors, respectively, shows a conspicuous projection pattern from layer $2 / 3$ to layer $5 \mathrm{~A}$ (Lübke et al., 2003) and from layer 5A to layer 2/3, respectively, in a vertically oriented and almost columnrestricted way, and thus reciprocal connectivity between these layers has been suggested (Bernardo et al., 1990; Gottlieb and Keller, 1997; Kim and Ebner, 1999). Underneath layer 1, the vertically projecting axon collaterals of L5A pyramidal cells bend and spread tangentially within and between adjacent cortical columns. This axonal projection of L5A pyramidal cells into layer 1 to, so far unidentified, target cells suggests, in conjunction with the axonal projection pattern of $\mathrm{L} 2 / 3$ pyramidal cells to layer $5 \mathrm{~A}$, the existence of a feedback loop between layer $2 / 3$ and layer $5 \mathrm{~A}$ (The L5A-to-L2/3 connection is established by Feldmeyer, unpublished results). Such a feedback loop could also exist between layer 4 and 5A; however, L5A-to-L4 connections have not been established in this study. Infragranular axon collaterals of $\mathrm{L} 2 / 3$ pyramidal cells innervate and excite the L5A pyramidal cells. The back projection of L5A pyramidal cells into layers $2 / 3$ and 1 then could excite L2/3 pyramidal cells in their basal and apical oblique dendrites, giving rise to intracortically generated oscillations presumably depending on appropriately timed inhibition (SanchezVives and McCormick, 2000).

\section{L5A output to other cortical areas: motor responses and attention}

L5A pyramidal cells project intracortically to the motor cortex and the secondary somatosensory cortex (Donoghue and Parham, 1983; Koralek et al., 1990). L5A pyramidal cells in the somatosensory cortex have the lowest threshold for eliciting a behavioral response by local extracellular electrical stimulation (Krauss, 2003). Thus, the AP pattern in L5A pyramidal cells could dominate the output from the somatosensory cortex that is conveyed to layers 5 and 6 of the motor cortex (M1) and there initiates or modulates whisker movements (Brecht et al., 2004). Possibly, the pattern of APs of L5A pyramidal cells is conveyed also to the secondary somatosensory cortex in helping to focus attention (Cauller and Kulics, 1991). Interestingly, L2/3 pyramidal cells project to the $\mathrm{M} 1$, but, because of the longer latency of APs generated in layer $2 / 3$, the motor response should be dominated by L5A output. Thus, because of their direct innervation by L4 spiny neurons and their albeit sparse projections to the motor cortex (Donoghue and Parham, 1983), these L5A pyramidal cells may constitute a rapid and major link between somatosensory and motor cortex attributable to their high rate and synchrony of APs.

\section{References}

Ahissar E, Kleinfeld D (2003) Closed-loop neuronal computations: focus on vibrissa somatosensation in rat. Cereb Cortex 13:53-62.

Ahissar E, Sosnik R, Haidarliu S (2000) Transformation from temporal to 
rate coding in a somatosensory thalamocortical pathway. Nature 406:302-306.

Ahissar E, Sosnik R, Bagdasarian K, Haidarliu S (2001) Temporal frequency of whisker movement. II. Laminar organization of cortical representations. J Neurophysiol 86:354-367.

Alloway KD, Crist J, Mutic JJ, Roy SA (1999) Corticostriatal projections from rat barrel cortex have an anisotropic organization that correlates with vibrissal whisking behavior. J Neurosci 19:10908-10922.

Armstrong-James M, Fox K (1987) Spatiotemporal convergence and divergence in the rat S1 "barrel" cortex. J Comp Neurol 263:265-281.

Bernardo KL, McCasland JS, Woolsey TA, Strominger RN (1990) Local intra- and interlaminar connections in mouse barrel cortex. J Comp Neurol 291:231-255.

Brecht M, Sakmann B (2002) Dynamic representation of whisker deflection by synaptic potentials in spiny stellate and pyramidal cells in the barrels and septa of layer 4 rat somatosensory cortex. J Physiol (Lond) 543:49-70.

Brecht M, Roth A, Sakmann B (2003) Dynamic receptive fields of reconstructed pyramidal cells in layers 3 and 2 of rat somatosensory barrel cortex. J Physiol (Lond) 553:243-265.

Brecht M, Schneider M, Sakmann B, Margrie TW (2004) Whisker movements evoked by stimulation of single pyramidal cells in rat motor cortex. Nature 427:704-710.

Castro-Alamancos MA (2004) Dynamics of sensory thalamocortical synaptic networks during information processing states. Prog Neurobiol 74:213-247.

Cauller LJ, Kulics AT (1991) The neural basis of the behaviorally relevant N1 component of the somatosensory-evoked potential in SI cortex of awake monkeys: evidence that backward cortical projections signal conscious touch sensation. Exp Brain Res 84:607-619.

Chmielowska J, Carvell GE, Simons DJ (1989) Spatial organization of thalamocortical and corticothalamic projection systems in the rat SmI barrel cortex. J Comp Neurol 285:325-338.

Crandall JE, Korde M, Caviness Jr VS (1986) Somata of layer V projection neurons in the mouse barrelfield cortex are in preferential register with the sides and septa of the barrels. Neurosci Lett 67:19-24.

Donoghue JP, Parham C (1983) Afferent connections of the lateral agranular field of the rat motor cortex. J Comp Neurol 217:390-404.

Feldmeyer D, Egger V, Lübke J, Sakmann B (1999) Reliable synaptic connections between pairs of excitatory layer 4 neurones within a single "barrel" of developing rat somatosensory cortex. J Physiol (Lond) 521:169-190.

Feldmeyer D, Lübke J, Silver RA, Sakmann B (2002) Synaptic connections between layer 4 spiny neurone-layer $2 / 3$ pyramidal cell pairs in juvenile rat barrel cortex: physiology and anatomy of interlaminar signalling within a cortical column. J Physiol (Lond) 538:803-822.

Gottlieb JP, Keller A (1997) Intrinsic circuitry and physiological properties of pyramidal neurons in rat barrel cortex. Exp Brain Res 115:47-60.

Huang W, Armstrong-James M, Rema V, Diamond ME, Ebner FF (1998) Contribution of supragranular layers to sensory processing and plasticity in adult rat barrel cortex. J Neurophysiol 80:3261-3271.

Kim U, Ebner FF (1999) Barrels and septa: separate circuits in rat barrels field cortex. J Comp Neurol 408:489-505.

Koralek KA, Jensen KF, Killackey HP (1988) Evidence for two complementary patterns of thalamic input to the rat somatosensory cortex. Brain Res 463:346-351.

Koralek KA, Olavarria J, Killackey HP (1990) Areal and laminar organiza- tion of corticocortical projections in the rat somatosensory cortex. J Comp Neurol 299:133-150.

Krauss A (2003) Psychophysische Untersuchung zur Detektierbarkeit elektrisch evozierter Aktivität in der somatosensorischen Hirnrinde der wachen Ratte. Diploma thesis, University of Heidelberg.

Lu SM, Lin RC (1993) Thalamic afferents of the rat barrel cortex: a lightand electron-microscopic study using Phaseolus vulgaris leucoagglutinin as an anterograde tracer. Somatosens Mot Res 10:1-16.

Lübke J, Egger V, Sakmann B, Feldmeyer D (2000) Columnar organization of single and synaptically coupled excitatory spiny neurons in layer 4 of the rat barrel cortex. J Neurosci 20:5300-5311.

Lübke J, Roth A, Feldmeyer D, Sakmann B (2003) Morphometric analysis of the columnar innervation domain of neurons connecting layer 4 and layer 2/3 of juvenile rat barrel cortex. Cereb Cortex 13:1051-1063.

Manns ID, Sakmann B, Brecht M (2004) Sub- and suprathreshold receptive field properties of pyramidal neurones in layers $5 \mathrm{~A}$ and $5 \mathrm{~B}$ of rat somatosensory barrel cortex. J Physiol (Lond) 556:601-622.

Markram H, Wang Y, Tsodyks M (1998) Differential signaling via the same axon of neocortical pyramidal neurons. Proc Natl Acad Sci USA 95:5323-5328.

Mercier BE, Legg CR, Glickstein M (1990) Basal ganglia and cerebellum receive different somatosensory information in rats. Proc Natl Acad Sci USA 87:4388-4392.

Moore CI, Nelson SB (1998) Spatio-temporal subthreshold receptive fields in the vibrissa representation of rat primary somatosensory cortex. J Neurophysiol 80:2882-2892.

Porter JT, Johnson CK, Agmon A (2001) Diverse types of interneurons generate thalamus-evoked feedforward inhibition in the mouse barrel cortex J Neurosci 21:2699-2710.

Reyes A, Lujan R, Rozov A, Burnashev N, Somogyi P, Sakmann B (1998) Target-cell-specific facilitation and depression in neocortical circuits. Nat Neurosci 1:279-285.

Rozov A, Burnashev N, Sakmann B, Neher E (2001) Transmitter release modulation by intracellular $\mathrm{Ca}^{2+}$ buffers in facilitating and depressing nerve terminals of pyramidal cells in layer $2 / 3$ of the rat neocortex indicates a target cell-specific difference in presynaptic calcium dynamics. J Physiol (Lond) 531:807-826.

Sanchez-Vives MV, McCormick DA (2000) Cellular and network mechanisms of rhythmic recurrent activity in neocortex. Nat Neurosci 3:1027-1034

Schubert D, Kötter R, Zilles K, Luhmann HJ, Staiger JF (2003) Functional input connectivity of layer VA pyramidal cells in rat barrel cortex. Sixth IBRO World Congress of Neuroscience, p 424. Prague, Czech Republic.

Swadlow HA (2003) Fast-spike interneurons and feedforward inhibition in awake sensory neocortex. Cereb Cortex 13:25-32.

Tarczy-Hornoch K, Martin KAC, Stratford KJ, Jack JJB (1999) Intracortical excitation of spiny neurons in layer 4 of cat striate cortex in vitro. Cereb Cortex 9:833-843.

Wirth C, Lüscher HR (2004) Spatiotemporal evolution of excitation and inhibition in the rat barrel cortex investigated with multielectrode arrays. J Neurophysiol 91:1635-1647.

Zhu Y, Stornetta RL, Zhu JJ (2004) Chandelier cells control excessive cortical excitation: characteristics of whisker-evoked synaptic responses of layer $2 / 3$ nonpyramidal and pyramidal neurons. J Neurosci 24:5101-5108. 